\title{
The closed loop military maintenance workforce: A simulation-based optimisation approach
}

\author{
$\underline{\text { Kurt R. Withers }}^{\text {a }}$, Hasan Hüseyin Turan ${ }^{\text {b }}$ (D), Sanath Kahagalage ${ }^{\text {b (D) , and Sondoss El Sawah }}{ }^{\text {b }}$ \\ ${ }^{a}$ The University of New South Wales at the Australian Defence Force Academy \\ ${ }^{\mathrm{b}}$ Capability Systems Centre, University of New South Wales, Canberra, Australia \\ Email: $\underline{\text { k.withers@student.unsw.edu.au }}$
}

\begin{abstract}
Workforce simulation modelling allows organisations to predict workforce skill requirements and implement strategies to meet foreseeable industry demands. The maintenance workforce increases the simulation model's complexity due to the co-dependencies associated with variables such as asset reliability, workforce composition, and maintenance policies. The military maintenance workforce differs from the usual maintenance workforce model due to its unique closed loop environment, which adds further complexities to the simulation model.

This paper uses a typical Australian Defence Force (ADF) technical workshop to explore the effect that workforce composition and posting policies have on a workshop's ability to maintain critical asset in a ready for issue (RFI) state. As the solution approach, a state-of-art Discrete Event Simulation (DES) model is coupled with a genetic algorithm (GA), which is known as simulation-based optimisation, to find best strategical decisions regarding posting and workforce composition in the technical workshop within the defence environment.
\end{abstract}

The objective of the DES is to explore the effects of utilising different posting policies and workforce compositions in a ADF technical workshop. We first assume that the asset transfer and time between asset transfer are not optimised for the associated workshop workforce. This simulates a situation in which an ADF workforce has been stood up and asset transfer is set on mission requirements rather than maintenance considerations. This phase contains posting policies of a purely military workshop for a 2 , 3 , and 4 year period. Next, the workforce used for a 2 year posting policy is altered and members are replaced with Australian Public Service (APS) staff. The primary focus of this work is the use of simulation-based optimisation to optimise the asset transfer amount and rate in order to improve work-

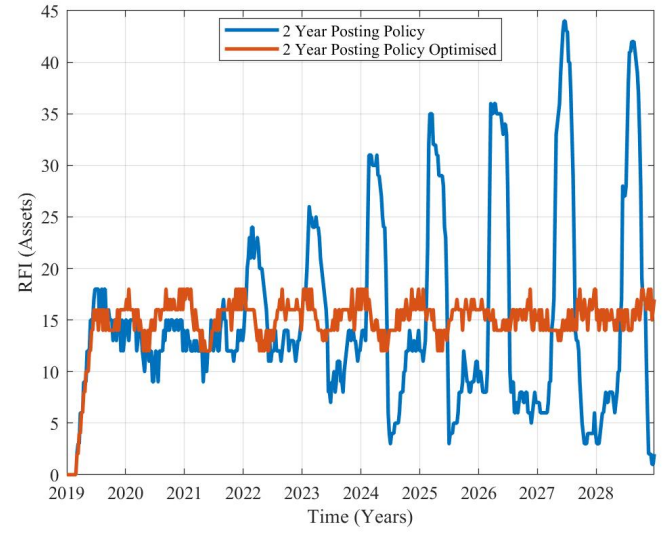

Figure 1. Effect of simulation-optimisation on the number of RFI assets. shop mean and range for assets in RFI state.

The findings reveal that a mixed workforce composition provides greater workshop flexibility. There is up to a $10 \%$ increase in the mean RFI asset availability and a 55\% decrease in the RFI variance when a mixed workforce composition is utilized. Further, simulation-based optimization leads to $9 \%$ mean increase and $80 \%$ variance reduction in the number of RFI assets compared to unoptimised results. The purely military workshop 2 year posting policy results are shown in Figure 1.

Keywords: Defence, maintenance, workforce composition, posting cycle, simulation-based optimisation, genetic algorithm 
K. R. Withers et al., The closed loop military maintenance workforce: A simulation-based optimisation approach.

\section{INTRODUCTION}

Workforce simulation has allowed organisations to predict future industry needs and implement strategies to ensure their organisation remains viable into the future. A workforce model incorporating maintenance requires an increased number of variables in areas of assets, resources, and a trained workforce. This is important for organisations that are reliant on a trained workforce to facilitate maintenance of critical assets. A key component in this workforce is the ability to either outsource work or draw from external providers when staffing and training deficiencies are found. This assumption allows the simplification of the workforce model; however, an open loop model allowing for workforce retention, recruitment, and training is not usable in the military application.

The military organisation is a closed loop system meaning that the workforce is dependent on constraints internal to the organisation (Turan et al. 2019). These constraints include, but are not limited to, internal promotion, internal training, and asset reliance for training. Incorrectly accounting for these can lead to an under-utilised workforce, loss of technical mastery, reduction in asset availability, and critical asset shortages.

Whilst there exists some academic literature surrounding workforce optimisation, there is a sparse amount of analysis conducted on military workforce's that incorporate critical asset maintenance. There is even less investigation on the use of simulation modelling and optimisation techniques to explore military related workforce dependencies in the maintenance environment. Two critical variables for analysis, posting policies and workforce compositions can affect workforce utilisation and mission critical asset availability.

The rest of the paper is organised as follows. Section 2 reviews the literature on workforce planning with focus on simulation modelling. Section 3 discusses the unique problems related to ADF technical workshops encompassing the closed loop environment. Section 4 summarises both the discrete event simulation (DES) and the genetic algorithm (GA) coupling process (i.e., simulation-based optimisation) used within this paper. Lastly, Section 5 summarises the results of DES model and simulation-based optimisation.

\section{Literature REVIEW}

Workforce modelling is primarily used in organisations that have complex work rules. Models effectively capture the workplace process to create a relationship that adheres as close as possible to the actual workforce (Bangsow 2012). These models allow complex organisations to identify potential workforce gaps in future requirements (Safarishahrbijari 2018). The goal is to vary the input variables for a specific outcome, done via running the simulation for all possible input combinations. Due to time constraints this is not feasible and instead the goal is to find optimal outputs for a set of specific input variables (Fu et al. 2015).

More complex workforce simulation models incorporate additional inter-dependencies of workforce capabilities in conjunction with asset availability (e.g., Ertogral \& Öztürk (2019), Tavakoli Kafiabad et al. (2021)). These include multi-skilled workforce in maintenance with workflows dependent upon asset availability and a trained workforce (Wang et al. 2020).

Maintenance and repairs of critical assets are crucial support activities to extend the lifetime of the assets and to ensure safe lifetime operation (Kobbacy \& Murthy 2008). To achieve this, a complex workforce simulation model is required that can support the many co-dependencies that a technical workforce relies on. These range from recruiting an aging workforce, on the job training (OJT) and the complexities that come with timing assets with maintenance windows and resource availability (Kafiabad et al. 2020).

The complexities of workforce simulations on rotatable mission critical assets is covered by Ertogral \& Öztürk (2019). This study utilised workforce simulation to show a constant workforce was able to reduce the overall organisation costs through early resource and asset sourcing policies. A key factor in Ertogral \& Öztürk (2019) findings were that the results relied heavily on a constant trained workforce which was able to utilise external sourcing when deficiencies were found. Another recent study focused on modelling railway network maintenance facilities proposed by Lidén et al. (2018) with the aim to model and optimise train (asset) availability which utilised asset free windows and a fixed set of maintainers. This workforce model was able to utilise workforce constraints to achieve maintenance in an efficient manner. Once again the workforce was assumed to be constant throughout the simulation and did not account for the loss of any trained staff. Lidén et al. (2018) assumed that any workforce deficiency was able be replaced with another equivalent trained externally sourced member.

Further studies have been undertaken into the complexities of training a sustainable workforce to ensure maintenance of critical equipment. For example, Tavakoli Kafiabad et al. (2021) utilised a two-stage stochastic 
model to obtain an optimal amount of maintainers to train to minimise the total workforce cost and late delivery fees. This study showed the importance of incorporating training of the workforce in a maintenance facility and the impact this has on late finishes against the total maintenance cost. The simulation model used did allow for outsourcing of maintenance and workforce borrowing strategies allowable in an open loop system.

The military technical workforce differs from other outside industries mainly due to the training system within the military environment where the experienced workforce is created. This differs from a civilian workplace as deficiencies can be recruited from outside the organisation. This means that military workforce deficiencies can have a large flow on effect to operational platforms or units. Analysis of literature surrounding workforce simulations within the military environment is limited. There exist some studies that concentrate on field specialisations. For example, Bastian et al. (2020) analysed the U.S Army cyber branch. In their study, the manning and training complexities mentioned above are considered; however, there is no interconnection with the workforce and any maintenance requirements. Horn et al. (2016) studied a workforce simulation planning problem to meet position requirements in the Australian Military environment. These papers once again fall short of integrating these models into a military maintenance facility to understand manning and posting policy impacts. The key point is there is limited literature available in the public domain that focuses on the complexities of a purely military workforce and the inter dependencies that a maintenance facility requires. Available papers concentrate on the ability of a specific stream to maintain a fully manned workforce; however, no consideration appears to be focused on how the military maintenance workforce capabilities and planning effect the ability of a workshop to conduct required mission critical asset maintenance.

\section{Problem definition}

A typical military maintenance workshop consists of several different ranked members as displayed in Figure 2. The lowest level member conducts the core business of the workshop with the higher ranked members conducting the inspecting of task progressions or additional administrative work related to noncore business. This has two primary effects that require consideration. The first is that the highest members usually have the least utilisation associated to core tasks. The second is that the junior members are the predominate contributors to the effectiveness of the workshop. The complication is that they are usually less skilled in the core workshop business and require OJT to become competent and usable within the workforce. While the junior members contribute the most to the workshop's effectiveness, they also are associated with

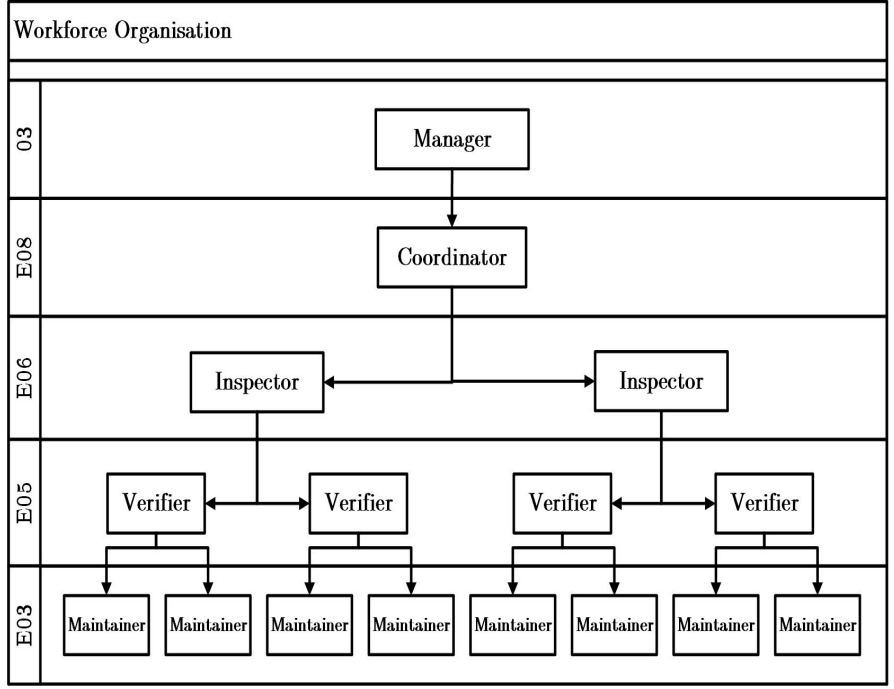

Figure 2. Workforce structure in the developed simulation model the largest impact of workshop effectiveness due to OJT on replacement.

OJT needs to be considered for the closed loop military workforce. Each member within the workforce will be posted and replaced with another member who requires OJT approximately every two years. This means the ability of a workshop to meet maintenance requirements is diminished until the member is competent. OJT is also dependant on availability of assets, there is the potential for unskilled staff to not be able to undertake the required OJT due to the availability of assets requiring maintenance. Both dependencies can lead to the loss of technical mastery within the workforce having greater flow on effects to the organisation.

For a purely military force, there exist time within the working day that the workforce must devote to non core business. This in turn effects the ability for a member to be utilised within the workshop environment. For the junior members, these non core maintenance tasks are usually associated with military specific training. This can result in an approximate $75 \%$ maximum utilisation of junior members to core business maintenance. This is necessary to account for accurate workshop simulation in the military environment.

A limited number of workshops within the military environment have a workforce composition containing non 
military staff. These are often found in specialised areas and in most cases the non military staff consist of Australian Public Service (APS) staff who are Subject-matter experts (SMEs) in their associated field. The APS workforce is not constricted by the closed loop military workforce requirements and demands and can remain as a constant source of experience. They are also recruited and trained interdependent of the military and provide greater flexibility for workforce replacement.

To investigate the effects that differing posting cycles and workforce composition have on a military workshop a DES model will be utilised to run two different sets of scenarios before using simulation-based optimisation. The first set of scenarios will consist of a purely military workshop utilising a number of posting policies. The second set of scenarios will consist of a mixed workforce composition utilising a 2 year posting policy. Both scenario sets will utilise a specific amount of critical assets being delivered at set intervals. The DES will then be coupled with optimisation using GA and all scenarios rerun with the amount of assets delivered and delivery schedule to the workshop being optimised to reduce mean and range of RFI assets.

\section{DETAILS OF SIMULATION MODEL AND SIMULATION-BASED OPTIMISATION}

\subsection{Simulation model}

The higher-level DES model is shown in Figure 3 and is run at hourly time steps. The model can be seen as two separate interacting sections. The first consists of the workflow for the maintenance with all assets starting within the storage facility requiring maintenance. At specific timings a number of assets are transferred to the workshop at a set rate and the competent workforce is checked for availability to conduct maintenance. When maintainers are available, they are removed from the pool of maintainers and the untrained workforce checked and assigned if available. On completion the asset is returned to the storage facility in a RFI state until its maintenance period has elapsed.

The second workflow consists of accounting for the posting policy of incoming members and OJT for untrained mem-

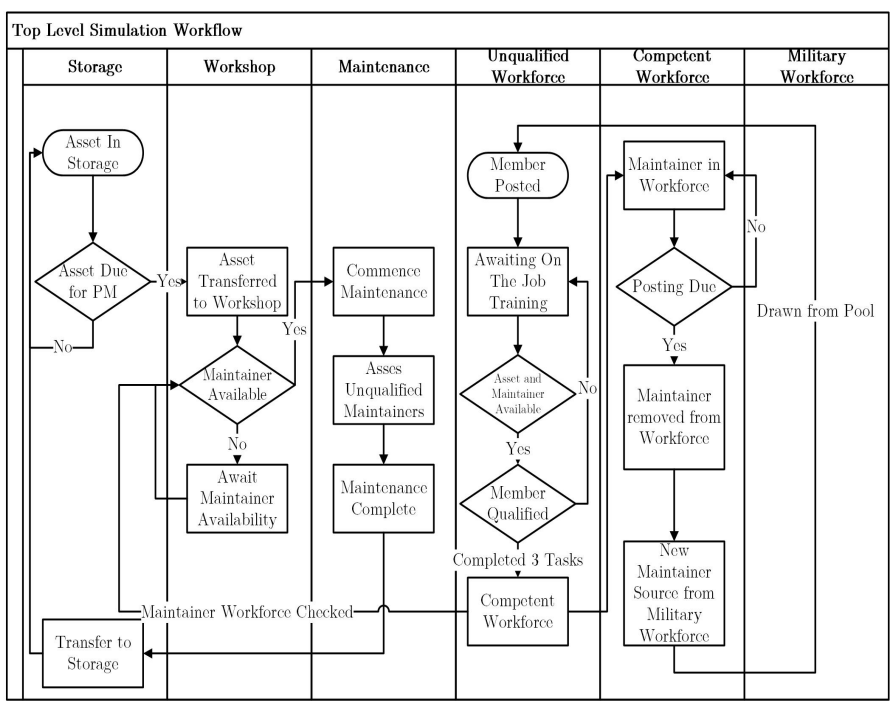

Figure 3. Top level visualization of the DES model bers to be deemed as competent. While a member is deemed as competent, they will be available to conduct maintenance as assets become available dependant on their maximum utilisation variable. After a designated posting period the competent member is replaced with an untrained member to simulate a posting cycle. OJT of untrained members requires an asset available for maintenance in addition to a competent member able to supervise. Once the maintenance of the asset is complete the untrained member is checked for the required number of competencies. If this criterion is meet, the member is moved to the competent workforce. If not the member remains unqualified awaiting further training. APS workshop staff are treated as nonmilitary staff and as such will be available for all work hours at maximum $90 \%$ utilisation. They do not suffer from posting and are assumed competent for the entirety of the simulation length. It is also assumed that during the length of the simulation the APS members do not separate from the workforce.

\subsection{Simulation-based optimisation}

On completion of the first scenario sets GA is coupled with the DES, as shown in Figure 4, in order to reduce the range and increase the mean of the RFI assets for each posting policy and workforce composition. The GA creates an initial population of individuals to analyse the DES output and assign a fitness value for each individual. This fitness value is used to evaluate which individual will be more likely to reproduce. In this way individuals within the population with less fitness are more likely to be replaced for the next generation. Selected parents are chosen for crossover and mutation before reassembling the population in order to create 
another generation to provide improved results (Luke 2013). In this way, the population is slowly altered until a desired parameter has been meet.

An individual is created with the assets per event and events per year converted into a binary representation in order to obtain an initial population of individuals. Each individual is converted into the associated integer value and utilised within the simulation run for the selected posting policy or workforce composition. On completion, the mean and range of the RFI assets are utilised in order to assign a fitness value for the associated individual of the population. This fitness value is utilised to evaluate the individual and measure its viability for crossover and mutation in future generations.

A tournament selection is then utilised on the population to select individuals for reproduction and further genetic modification. This utilises a function in which individual values are chosen at random from within the population and compared, individuals with higher fitness values are more likely to be selected as the winner. In this way, even a candidate with a lower fitness value maybe selected for further crossover and mutation which may yield a higher fitness value in future generations.

A one point crossover function is applied to two randomly selected parents to produce offspring. This function selects one bit within both parents and designates that point as the crossover point. All bits to the right of the selected point in both parents are then swapped. The resultant is two offspring that each contain some part of the original information from the parents (Luke 2013).

After crossover, a mutation function is applied to individuals selected at random. The mutation function

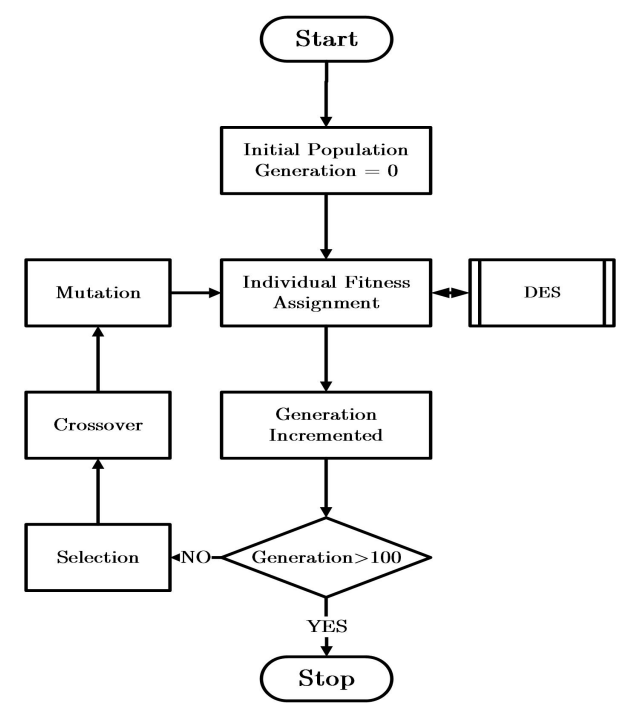

Figure 4. Top Level Visualization of the GA Model uses a low probability that an arbitrary bit within the individual will be flipped from its original state. This operation generally ensures that populations are not caught in local maximums/minimums and explore further individuals that may find the global maximum/minimum (Turan et al. 2020).

GA was coded with the assistance of the DEAP python tool set (Fortin et al. 2012). An initial population size of 50 was chosen with a generation limit of 100 . The crossover and mutation probability were set at 0.5 and 0.2 respectively. With the chance of a selected bit within the individual being mutated at 0.15 . The average run time for each selected posting policy or workforce composition was approximately 42 hours on a standard desktop computer with 12 cores and a $2.10 \mathrm{GHz}$ CPU.

\section{Computational analysis}

Figure 5 displays the results of a 10 year simulation for a ADF technical workshop utilising a 2, 3 or 4 year posting policy. Figure 6 displays the results of a 10 year simulation with a workforce composition of either entirely military workforce or mixed workforce of 1 Verifier and 2 Maintainers as APS or 2 Verifiers and 4 Maintainers as APS. Figure 2 can be utilised for greater clarity of mixed workforce composition. The tabulated mean and range of these results are shown in Table 1 with \% increase or decrease referencing the purely military workforce under a two year posting policy.

Table 1. Tabulated Results For Simulation

\begin{tabular}{ccccccc}
\hline Posting Policy & Assets Per Event & Events per Year & RFI Mean & RFI Range & Mean Increase (\%) & Range Decrease (\%) \\
\hline 2 & 3 & 30 & 15.1 & 40 & 0 & 3 \\
3 & 3 & 30 & 15.5 & 31 & 23 & 7 \\
4 & 3 & 30 & 16.1 & 20 & 0 & 50 \\
\hline Workforce Composition & Assets Per Event & Events per Year & RFI Mean & RFI Range & Mean Increase (\%) & Range Decrease (\%) \\
\hline All Military & 3 & 30 & 15.1 & 40 & 0 & 0 \\
3 APS & 3 & 30 & 15.6 & 27 & 4 & 33 \\
6 APS & 3 & 30 & 16.5 & 18 & 10 & 55 \\
\hline
\end{tabular}


K. R. Withers et al., The closed loop military maintenance workforce: A simulation-based optimisation approach.

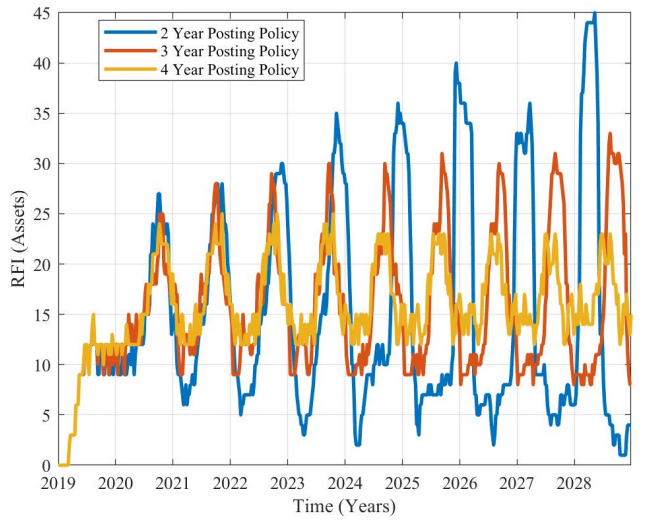

Figure 5. RFI Stores for a 2, 3 and 4 Year Posting Cycle

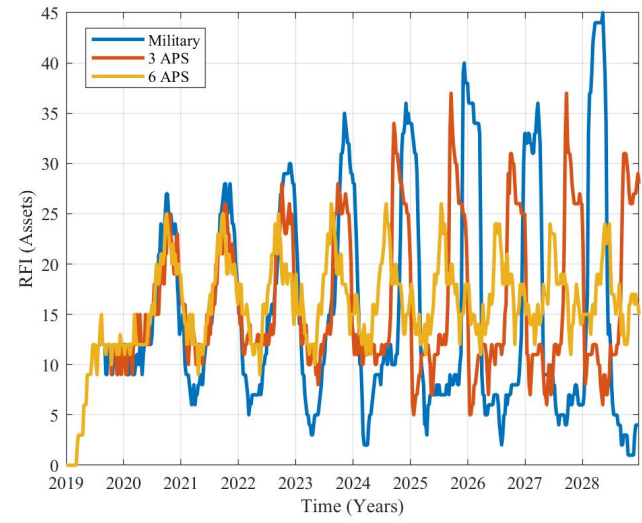

Figure 6. RFI Stores for Selected Workforce Compositions

Figure 7 shows the results utilising a GA optimisation for asset injection for a 2, 3, and 4 year posting policy. Figure 8 displays the results utilising a GA optimisation for asset injection for a ADF technical workshop with a workforce composition explained previously. The tabulated mean and range of these results are shown in Table 2 with $\%$ increase or decrease referencing the unoptimised scenario results show in Table 1

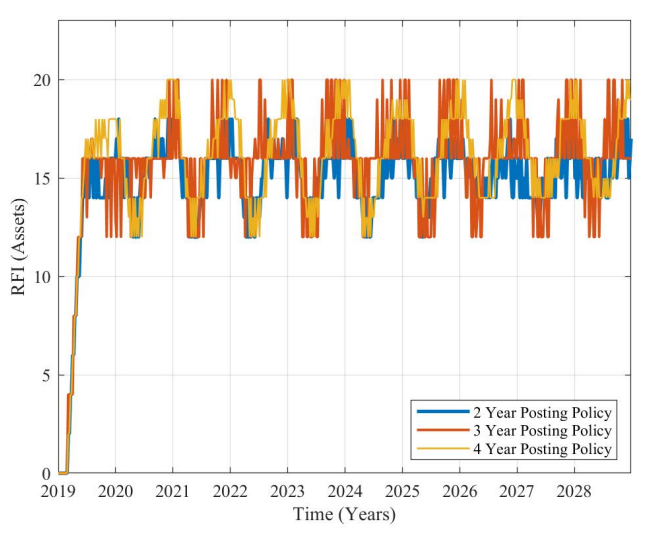

Figure 7. RFI Stores for Posting Policies with Optimised Asset Injection

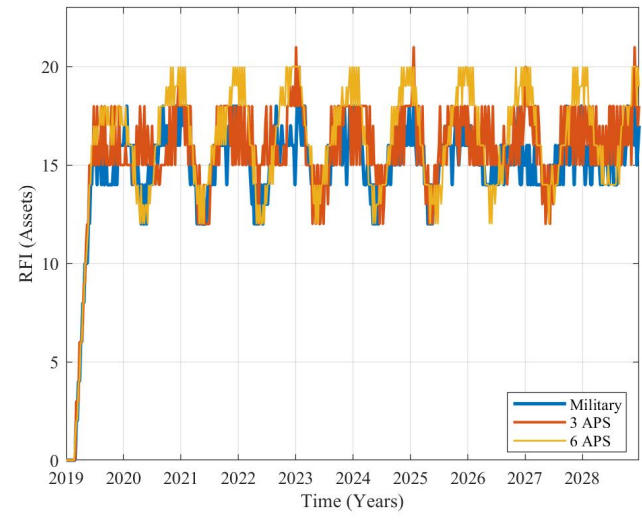

Figure 8. RFI Stores for Workforce Compositions with Optimised Asset Injection

Table 2. Tabulated Results For Simulation-based Optimisation

\begin{tabular}{|c|c|c|c|c|c|c|}
\hline Posting Policy & Assets Per Event & Events per Year & RFI Mean & RFI Range & Mean Increase (\%) & Range Decrease (\%) \\
\hline 2 & 2 & 24 & 15.2 & 9 & 1 & 78 \\
\hline 4 & 2 & 25 & 16.3 & 8 & 1 & 60 \\
\hline All Military & 2 & 24 & 15.2 & 9 & 1 & 78 \\
\hline 3 APS & 3 & 16 & 16 & 8 & 3 & 70 \\
\hline 6 APS & 2 & 25 & 16.5 & 8 & 0 & 56 \\
\hline
\end{tabular}

The results indicate that when asset injection is not optimised, an extended posting policy has a $7 \%$ RFI mean increase and a $50 \%$ range decrease over a 10 year period. This is due to the decreased down time of untrained staff to OJT requirements. When comparing a mixed technical workforce, swapping $25 \%$ of the E03s and E02s results in a RFI mean and range comparable to extending the workshops posting cycle by a one year period. The same can also be said by replacing $50 \%$ of the workshop workforce, this has a comparable effect of extending the 2 year posting to a 4 year posting. When asset transfer cannot be aligned to workshop composition, extending time between postings will allow for improved asset maintenance consistency. If the operation allowed, incorporation of non military staff would also see beneficial results comparable to those of extending a military members posting.

The increase to RFI mean by extending posting policies or replacing members with APS staff can be con- 
K. R. Withers et al., The closed loop military maintenance workforce: A simulation-based optimisation approach.

tributed to reduced OJT. As a posting policy is extended, there are fewer hours accounted to the untrained workforce and more hours associated to the trained workforce resulting in an increased RFI mean. The reduction in range is due to the reduced OJT requirements, this results in a more trained workforce able to conduct the required steps for asset planned maintenance completion at the scheduled delivery maintenance windows. Thus more consistent completion of assets rather than creating a backlog of assets that have their associated PM tasks completed in conjunction with other asset delivery windows.

The results also show that if asset injection can be considered, despite the choice of posting policy or workforce composition, the results of the workshops RFI stores output is comparable from scenario to scenario. The major difference between scenarios is a possibility for a $7 \%$ increase is RFI assets. The consistent results are due to the asset availability being injected in such a way that competent staff are available to conduct OJT when both assets and untrained staff are available. The asset maintenance windows also accounts for any hours required for the untrained workforce to become competent and reduces backlogs of assets during times when untrained staff may be at a maximum. The additional benefit seen in optimisation is that approximately $45 \%$ less assets are required in the optimised scenarios for similar mean outputs seen in the uncoupled DES model.

\section{CONCLUSIONS AND FURTHER RESEARCH DIRECTIONS}

The ADF technical workshop operates within a closed loop environment. It is required to grow members internally and cannot easily replace lost members that are posted regularly. Additionally, the technical mastery required to work on critical assets means that junior workshop members are not competent when first posted to a position and require OJT to transition to the component workforce. Through simulation it was shown that a mixed workforce containing Military and APS members provides greater flexibility increasing RFI stores output by $10 \%$ and reducing the overall asset range by $55 \%$. Should this not be available it was shown that extending the posting period to 4 years results in a mean increase of $7 \%$ and a range reduction of $50 \%$.

GA optimisation coupling was used in order to match assets transferred and the yearly transfer rate to the simulated workforce. It was shown that each workforce was capable of producing a similar mean, with a maximum difference between these means of $7 \%$. All scenarios saw a range reduction of up to $80 \%$ when compared to the worst case unoptimised scenario. The additional benefit of GA coupling was a $45 \%$ reduction in the amount of critical assets to produce superior results.

As future research, it would be beneficial to use historical data for comparable real world examples. The DES and optimisation process is also expandable to remove assumptions, such as resources always being available for the planned maintenance, to be taken into account for future projects. Lastly, refinement of the simulation model for the direct application of this research would prove beneficial to the overall data obtained.

\section{REFERENCES}

Bangsow, S. (2012), 'Use cases of discrete event simulation', Appliance and research, Berlin, New York .

Bastian, N. D., Lunday, B. J., Fisher, C. B. \& Hall, A. O. (2020), 'Models and methods for workforce planning under uncertainty: Optimizing us army cyber branch readiness and manning', Omega $\mathbf{9 2}, 102171$.

Ertogral, K. \& Öztürk, F. S. (2019), 'An integrated production scheduling and workforce capacity planning model for the maintenance and repair operations in airline industry', Computers \& Industrial Engineering 127, 832-840.

Fortin, F.-A., De Rainville, F.-M., Gardner, M.-A., Parizeau, M. \& Gagné, C. (2012), 'DEAP: Evolutionary algorithms made easy', Journal of Machine Learning Research 13, 2171-2175.

Fu, C., Fu, C. \& Michael, M. (2015), Handbook of simulation optimization, Springer.

Horn, M. E., Elgindy, T. \& Gomez-Iglesias, A. (2016), 'Strategic workforce planning for the australian defence force', Journal of the Operational Research Society 67(4), 664-675.

Kafiabad, S. T., Zanjani, M. K. \& Nourelfath, M. (2020), 'Integrated planning of operations and on-job training in maintenance logistics networks', Reliability Engineering \& System Safety 199, 106922.

Kobbacy, K. A. H. \& Murthy, D. P. (2008), Complex system maintenance handbook, Springer Science \& Business Media.

Lidén, T., Kalinowski, T. \& Waterer, H. (2018), 'Resource considerations for integrated planning of railway traffic and maintenance windows', Journal of rail transport planning \& management 8(1), 1-15.

Luke, S. (2013), Essentials of Metaheuristics, second edn, Lulu. Available for free at http://cs.gmu.edu/ sean/book/metaheuristics/.

Safarishahrbijari, A. (2018), 'Workforce forecasting models: a systematic review', Journal of Forecasting 37(7), 739-753.

Tavakoli Kafiabad, S., Zanjani, M. K. \& Nourelfath, M. (2021), 'Workforce training and operations planning for maintenance centres under demand uncertainty', International Journal of Production Research pp. 1-13.

Turan, H. H., Atmis, M., Kosanoglu, F., Elsawah, S. \& Ryan, M. J. (2020), 'A risk-averse simulation-based approach for a joint optimization of workforce capacity, spare part stocks and scheduling priorities in maintenance planning', Reliability Engineering \& System Safety 204, 107199.

Turan, H. H., Elsawah, S. \& Ryan, M. J. (2019), Simulation-based analysis of military workforce planning strategies, in 'Proceedings of the 2019 International Conference on Management Science and Industrial Engineering', pp. 68-75.

Wang, H., Alidaee, B., Ortiz, J. \& Wang, W. (2020), 'The multi-skilled multi-period workforce assignment problem', International Journal of Production Research pp. 1-18. 